\title{
The NPI cyclotron-based fast neutron facility
}

\author{
P. Bém ${ }^{1, a}$, V. Burjan ${ }^{1}$, J. Dobeš ${ }^{1}$, U. Fischer ${ }^{2}$, M. Götz ${ }^{1}$, M. Honusek ${ }^{1}$, V. Kroha ${ }^{1}$, J. Novák ${ }^{1}$, \\ S.P. Simakov², and E. Šimečková ${ }^{1}$ \\ ${ }^{1}$ Nuclear Physics Institute v.v.i., 25068 Řež, Czech Republic \\ ${ }^{2}$ Forschungszentrum Karlsruhe, Institut für Reaktorsicherheit, Postfach 3640, 76021 Karlsruhe, Germany
}

\begin{abstract}
For validating activation cross-section data in the energy range relevant to IFMIF (International Fusion Material Irradiation Facility), the fast neutron sources were developed utilizing the NPI variable-energy cyclotron U-120M. Novel concept of source reaction was investigated for a generation of the white - (IFMIF-like) - spectrum on NPI cyclotron. The deuteron break-up process induced by $37 \mathrm{MeV}$ protons on flowing heavy water target was found to produce neutrons with high intensity, mean energy of $14 \mathrm{MeV}$ and spectrum extending to $32 \mathrm{MeV}$. The small-size samples (diameter of $15 \mathrm{~mm}$ ) are irradiated at neutron flux up to $10^{11} \mathrm{n} / \mathrm{cm}^{2} / \mathrm{s}$ in this source. The quasi-monoenergetic neutron field in the energy range from 20 to $35 \mathrm{MeV}$ is produced using standard ${ }^{7} \mathrm{Li}(\mathrm{p}, \mathrm{n})$ reaction on thin (carbon backed) lithium foil. The p-Li source produces neutron flux up to $10^{9} \mathrm{n} / \mathrm{cm}^{2} / \mathrm{s}$.

The present work deals with methodical, technical and functional characteristics of $\mathrm{p}-\mathrm{D}_{2} \mathrm{O}$ and $\mathrm{p}-{ }^{7} \mathrm{Li}$ neutron sources. The determination of spectral flux at position of irradiated samples is described in details. A summary of present and next program of experiments is outlined.
\end{abstract}

\section{Introduction}

The concept of International Fusion Material Irradiation Facility (IFMIF) consists in high-power deuteron accelerator $(40 \mathrm{MeV})$ and lithium (liquid jet) target. The neutron source reaction $\mathrm{d}(40 \mathrm{MeV})+\mathrm{Li}$ produces a white spectrum with mean energy of $14 \mathrm{MeV}$ and a energy range up to $35 \mathrm{MeV}$ (weak tail up to $55 \mathrm{MeV}$ ). The calculations of neutronic responses in the components of IFMIF Test Cell need the activation-cross-sections (ACS) database, which is validated against experiments at relevant (IFMIF-like) energy range.

For integral benchmark experiments, the white-spectrum neutron source was developed at NPI using the variableenergy cyclotron U-120 M. To simulate the neutron spectrum in the energy range relevant to IFMIF, the new source reaction $\mathrm{p}(37 \mathrm{MeV})+\mathrm{D}_{2} \mathrm{O}$ (thick target) has been investigated and high-power heavy-water-flow target was then developed. To determine the neutron spectral flux in the position of irradiated samples, the activation foil method was used. Present work deals mainly with the experimental verification of spectral flux in the vicinity of source target.

In the review on a status of nuclear data files [1], a number of elements relevant to IFMIF was identified for which neutron activation data are inadequate and new ACS measurements above $20 \mathrm{MeV}$ are needed. Therefore, in parallel to white-spectrum $\mathrm{p}-\mathrm{D}_{2} \mathrm{O}$ source, the quasimonoenergetic $\mathrm{p}-{ }^{7} \mathrm{Li}$ source with neutron energies between 20 to $37 \mathrm{MeV}$ has been build up.

The program of neutron activation experiments carried out at NPI within the IFMIF project and the EFDA Fusion Technology Programme is outlined as well.

${ }^{a}$ Presenting author, e-mail: bem@ujf.cas.cz

\section{White-spectrum $p-D_{2} O$ neutron source}

\subsection{Thick-target spectral yield of the $D_{2} O(p, x n)$ reaction}

Due to technical reasons, the powerful fast neutron sources are usually build-up using proton (deuteron) induced reactions on thick beryllium target. To avoid problems with an operation of toxic Be material, another light-nuclei targets were examined previously at NPI. In this investigation, high neutron emission from break-up of deuteron by fast light projectiles was indicated. The first-time investigated $\mathrm{D}_{2} \mathrm{O}(\mathrm{p}, \mathrm{xn})$ reaction was measured utilizing proton beams of the NPI variable energy cyclotron U-120M. The arrangement of neutron yield experiments and the data acquisition system have been described elsewhere [2]. The spectral yield data measured at $24 \mathrm{MeV}$

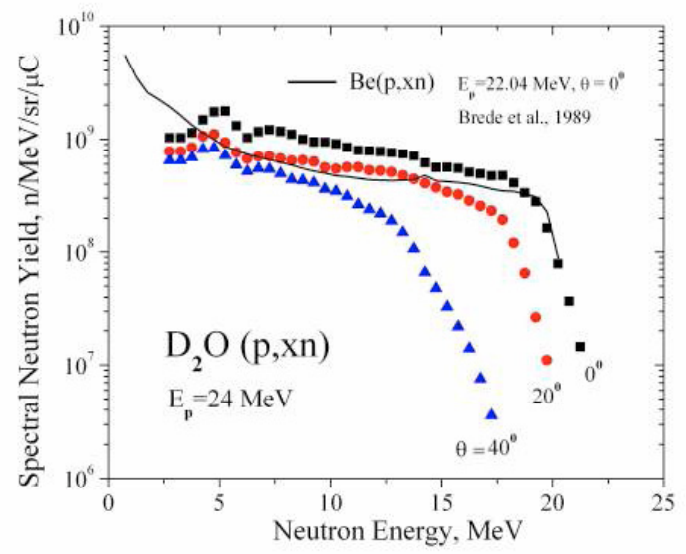

Fig. 1. Spectral neutron yield of neutrons from the $\mathrm{p}-\mathrm{D}_{2} \mathrm{O}$ reaction induced by of $24 \mathrm{MeV}$ protons. The zero- degree spectral yield from reaction on the $\mathrm{Be}$ target at $22 \mathrm{MeV}$ [3] is given for a comparison. 
proton energy are shown in figure 1 together with spectrum of the $\mathrm{Be}(\mathrm{p}, \mathrm{xn})$ reaction at $22 \mathrm{MeV}$ [3] given for comparison. The similar shape and slightly higher value of the spectral yield prove the $\mathrm{p}-\mathrm{D}_{2} \mathrm{O}$ reaction as suitable new fast neutron source.

The data at $37 \mathrm{MeV}$ energy were collected using the proton beam from negative-ion mode of acceleration. Resulting zerodegree spectral yield extends to neutron energy of $32 \mathrm{MeV}$ (see the fig. 3). Fluence-averaged mean neutron energy of spectrum is $13.9 \mathrm{MeV}$. Both observables well simulate the spectrum of the IFMIF source reaction $\mathrm{d}(40 \mathrm{MeV})+\mathrm{Li}$ which provides suitable tool for integral validation experiments relevant to the IFMIF neutronic calculations.

\subsection{High-power $\mathrm{D}_{2} \mathrm{O}$ target station}

The $\mathrm{D}_{2} \mathrm{O}$ target station was build up on the beam line of the cyclotron operated in the negative-ion mode of acceleration. In this mode, high proton beam power $(600 \mathrm{~W}$ for $37 \mathrm{MeV})$ and good beam-current stability present suitable basis for neutron activation experiments. To control the heat dissipation of proton beam, the flowing target medium with intensive external cooling has been selected and relevant assembly for flowing-medium target operation has been developed. The cell of $30 \mathrm{~mm}$ in diameter and $16 \mathrm{~mm}$ of length is equipped by $40 \mu \mathrm{m}$ thick entrance $\mathrm{Ta}$ foil $(0.5 \mathrm{MeV}$ thickness for the $37 \mathrm{MeV}$ proton beam).

The presence of bubbles in the target volume was observed as due to the non-laminar flow, the notable electro-dissociation and the micro-evaporation (caused by the beam power in the target). To avoid these phenomena, the inlet/outlet target apertures in form of slots were utilized. The drift velocity together with inlet/outlet pressure on cell were empirically adjusted. They are controlled by pressure-compensator system (argon gas) equipped by break bleeding valves and glass-level gauge remotely monitored by TV circuit.

The target cell together with the driving and cooling systems are insulated from the earth in such a way that proton current on the target can be accurately measured. The current of proton beam together with the drift, temperature and pressure of flowing water in topic parts of the system

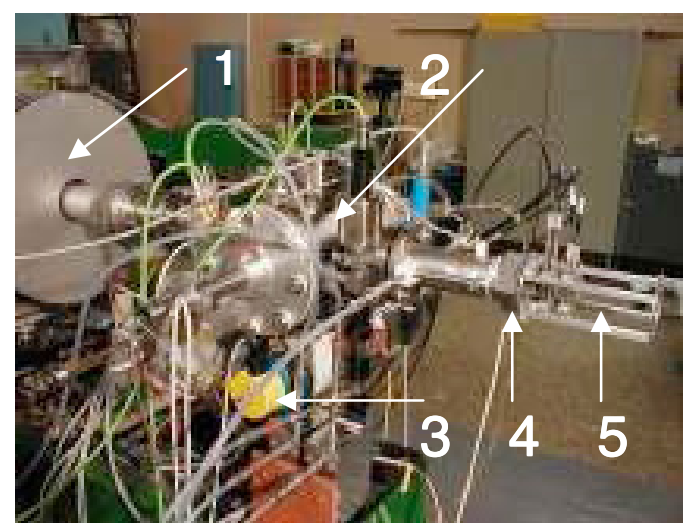

Fig. 2. Photograph of the $\mathrm{D}_{2} \mathrm{O}$-target station. (1) Quadrupole triplet of cyclotron-beam line, (2) beam diagnostics, (3) vacuum hardware, fallback- and pressure control system, (4) target chamber and (5) the holder for irradiated samples. are measured, digitized and registered by PC. In figure 2, a photograph of the $\mathrm{D}_{2} \mathrm{O}$-target station is given.

\subsection{Determination of spectral flux at sample positions}

Because of intensity reason, the irradiated samples are inserted very close to the source target. Due to the averaging of crosssection observables over sample- and target dimension, the spectral yield measured at large distance of the detector from target of source reaction ("point-like-geometry" arrangement of experiment) is not sufficient to determine the spectral flux at sample position.

As the most appropriate method, the multi-foil activation technique was utilized to determine the spectral neutron flux across the irradiated samples and to investigate the integration effects in details. A set of dosimetry foils Al, Ti, Fe, Co, Ni, $\mathrm{Y}, \mathrm{Nb}, \mathrm{Lu}$ and $\mathrm{Au}$ were irradiated by neutrons from the $\mathrm{p}-\mathrm{D}_{2} \mathrm{O}$ source at various distances from the target. Standard gammaspectrometry technique was employed for determination of induced $\gamma$-ray activities of reaction products. The reaction rates, specific- and saturated activities were calculated from measured data.

The full set of foils was irradiated at 3 and $156 \mathrm{~mm}$ distances. Up to 29 activation reactions were employed for the neutron spectra adjustment [4]. In the unfolding procedure, a modified form of SAND-II code was used (the nuclear data from EAF-2005 library for neutron energies to $55 \mathrm{MeV}$ were added). For the unfolding iterative procedure the initial guess neutron spectrum measured by scintillation unfolding technique (at $37 \mathrm{MeV}$ proton energy) was used.

The final spectrum obtained after adjustment procedure to data at $3 \mathrm{~mm}$ distance is shown in figure 3 (upper part) where the initial guess spectrum is also displayed. In the bottom part the calculated to measured saturated activities ratios $\mathrm{A}_{\text {cal }} / \mathrm{A}_{\exp }$ for dosimetry reactions used in present analyses are shown. The abscissa (E) means the energy weighted with product of neutron flux and corresponding cross section, the horizontal bars are the mean square deviations. In the energy range 10 to $25 \mathrm{MeV}$, the uncertainties of adjusted spectrum amounts $3 \%$. Above this range the uncertainty are estimated at the level of 20-30\% [8]. To achieve better knowledge of the spectrum below 10 and above $25 \mathrm{MeV}$ the (n,n') reactions on ${ }^{93} \mathrm{Nb}$, ${ }^{103} \mathrm{Rh}$ and ${ }^{115} \mathrm{In}$ and high threshold reactions ${ }^{209} \mathrm{Bi}(\mathrm{n}, \mathrm{xn})$ and are used in measurements being in progress.

In the figure 4 , the reaction rates of individual reaction products are set out as a ratio of data determined at 3 and $156 \mathrm{~mm}$ distance and displayed along the values E. The varying of neutron spectrum with distance is clearly indicated and experimentally proven. Within quoted uncertainties of data, the variance is equally well reproduced by the $1^{\text {st }}$ and $2^{\text {nd }}$ order polynomial form. The ratio of spectra resulted from SANDadjusting procedure shows some structure of this variance.

Some selected foil combinations were irradiated also at 47 , 57 , and $67 \mathrm{~mm}$ of s-t-d distance. In the figure 5, the rate values of reactions on foils at various distances are normalized to relevant data at longest distance. As the $\mathrm{X}$-axis, the distance $\mathrm{R}$ from the center of effective target thickness is accepted. Such representation enables in appropriate way the comparison of 


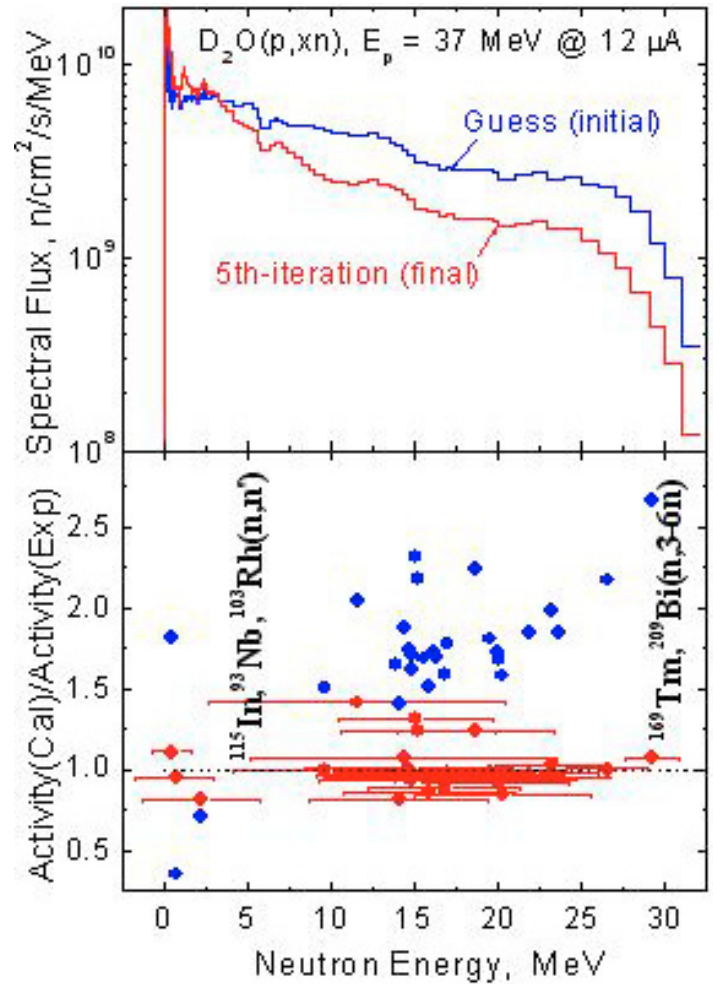

Fig. 3. Neutron flux and $\mathrm{C} / \mathrm{E}$ ratios obtained from the $\gamma$-activities of dosimetry foils by the SAND-II code: the guess (input) spectrum used for de-convolution and curves after corresponding iterations are signed.

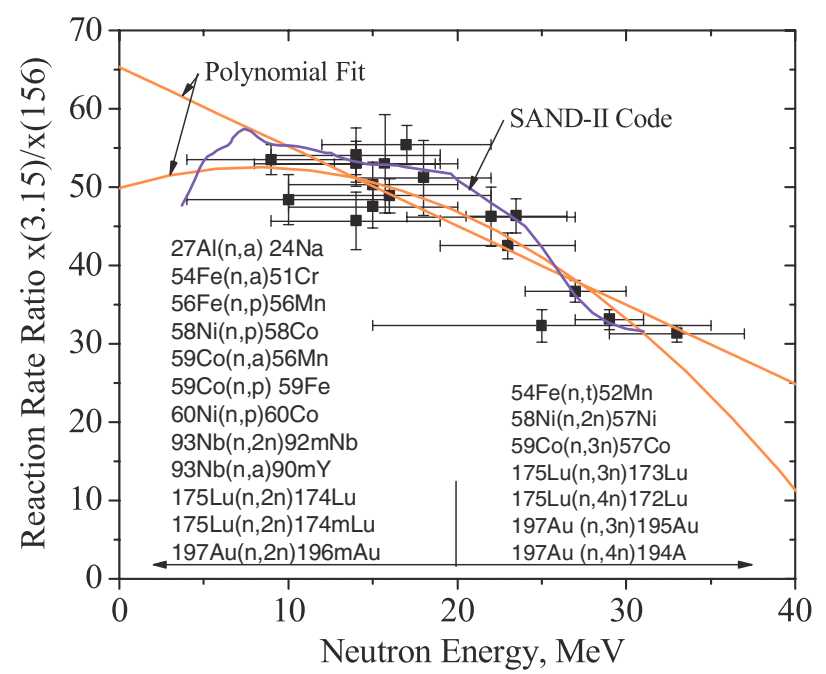

Fig. 4. Reaction rates ratio obtained from the irradiation of set of dosimetry foils set by white-spectrum neutron flux of the $\mathrm{p}(37 \mathrm{MeV})+\mathrm{D}_{2} \mathrm{O}$ source. For the further explanation see text.

measured reaction-rate ratio to the $1 / \mathrm{R}^{2}$ law. Data of reactions are grouped along the energy $\mathbf{E}$ (above and below $20 \mathrm{MeV}$ ).

The exponential fit was done through the data from low-threshold reaction ${ }^{27} \mathrm{Al}(\mathrm{n}, \alpha)$ (upper curve) and highestthreshold reactions ${ }^{54} \mathrm{Fe}(\mathrm{n}, \mathrm{t}),{ }^{59} \mathrm{Co}(\mathrm{n}, 3 \mathrm{n}),{ }^{175} \mathrm{Lu}(\mathrm{n}, 4 \mathrm{n})$, ${ }^{197} \mathrm{Au}(\mathrm{n}, 4 \mathrm{n})$ (lower curve). Besides the deviation of spectral flux from the $1 / \mathrm{R}^{2}$ law, slightly weaker variance of the spectral

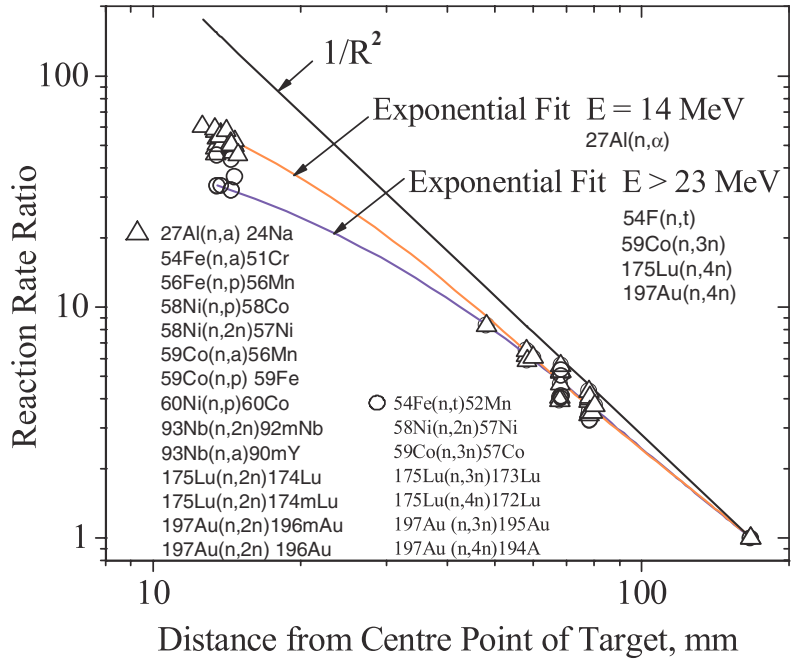

Fig. 5. Reaction rates ratio obtained from the irradiation of the dosimetry foils set by white-spectrum neutron flux of the $\mathrm{p}(37 \mathrm{MeV})+\mathrm{D}_{2} \mathrm{O}$ source. Data of individual reaction products are clustered in groups with $\mathbf{E}$ above and below $20 \mathrm{MeV}$ (circles and triangles, respectively). For the further explanation see text.

shape with distance is well seen. Using data at $3 \mathrm{~mm}$ distance and the quantitative form of observed averaging effects, the spectral flux at arbitrary distance could be determined with an accuracy of $10-15 \%$. Above $7 \mathrm{MeV}$ neutron energy, the flux $\mathrm{Y}$, amounts $5 \cdot 4^{10} \mathrm{n} / \mathrm{cm}^{2} / \mathrm{s}$ for the nearest distance from the target bottom and for $15 \mu \mathrm{A}$ proton beam current.

More detail experimental investigation of the spectrum at energies below $7 \mathrm{MeV}$ and above $25 \mathrm{MeV}$ is in progress using further low- and high-threshold dosimetry reactions, such as $\left(\mathrm{n}, \mathrm{n}^{\prime}\right)$ and $(\mathrm{n}, \mathrm{xn})$, respectively.

\section{Quasi-monoenergetic $\mathrm{p}-{ }^{7} \mathrm{Li}$ neutron source}

The standard ${ }^{7} \mathrm{Li}(\mathrm{p}, \mathrm{n})$ reaction on thin lithium target induced by $20-38 \mathrm{MeV}$ proton beam from the cyclotron is used for the production of quasi-monoenergetic neutron field. A selfsupporting Li target and the carbon beam stopper are utilized (the set-up similar to the design used at INS Tokyo [5]). Several foil/backing configurations and cooling $\left(5^{\circ} \mathrm{C}\right.$ spirit $)$ were investigated.

Finally, up to $400 \mathrm{~W} / \mathrm{cm}^{3}$ of beam power could be dissipated in $2 \mathrm{~mm} \mathrm{Li}$ foil under separate setting of the foil and carbon disc - see figure 6 (here, an overall view on the target station is also shown). The neutron flux of $6 \cdot 10^{8} \mathrm{n} / \mathrm{cm}^{2} / \mathrm{s}$ is calculated for $38 \mathrm{MeV} / 10 \mu \mathrm{A}$ proton beam and for the target-to-sample distance of $50 \mathrm{~mm}$. In the calculation, the cross-section data of ${ }^{7} \mathrm{Li}(\mathrm{p}, \mathrm{n})$ reaction from the ref. [5] are adopted. The independent investigation of spectra by protonrecoil-telescope technique is under way.

\section{Integral validation experiments}

Utilizing neutrons of IFMIF-like white-spectrum from the $\mathrm{p}-\mathrm{D}_{2} \mathrm{O}$ source, the integral activation data for IFMIF relevant 


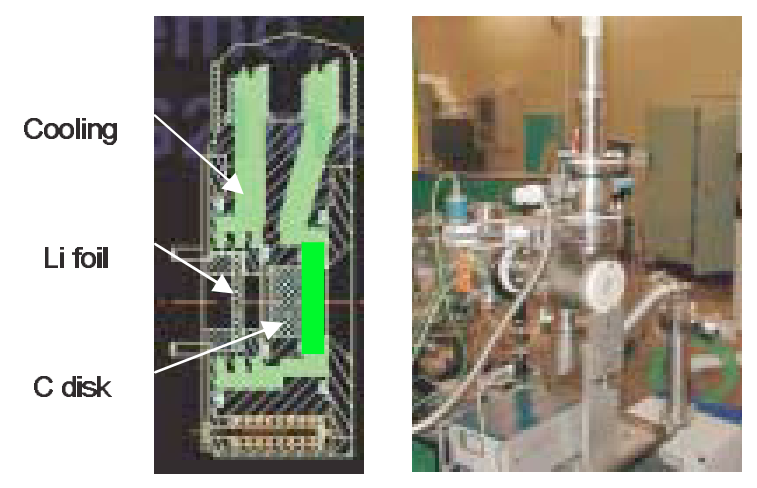

Fig. 6. The setup of the Li-target (on the left) and the sight on the Li-target station (on the right) are given. For the details see text.

materials (low-activation steel and its components) are collected and used to the validation of both the IEAF [1] and EAF [6] libraries. The integral validation experiments were carried out on Eurofer-97, Ta, W and $\mathrm{Cr}[6,7]$. These investigations serve also for identification the set of nuclides for which the differential-like validation experiments with the $\mathrm{p}$-7Li source needs to be done. Such program has been started by the investigation on $\mathrm{Ta}$ and $\mathrm{Cr}$ [8]. the experimental validation experiments on dosimetry-relevant nuclides at IMIF energies are under way $[4,6]$ as well.

\section{Conclusions}

The new high-power fast neutron source based on the $\mathrm{D}_{2} \mathrm{O}(\mathrm{p}, \mathrm{xn})$ reaction was developed for an integral validation of activation cross sections at IFMIF relevant energies. In a comparison with standard $\mathrm{p}$-Be source, the higher neutron yield, no toxic hazard and weaker limitation in incident beam intensity are advantages. More complicated target hardware needed to control a bubble-free flow of water presents some disadvantage. The spectral flux in the energy range of $7-$ $32 \mathrm{MeV}$ and its variance with source-to-target distance have been well determined by dosimetry-foil-method experiment backed by the MC calculations.

In parallel, the $\mathrm{p}^{7} \mathrm{Li}$ neutron source of quasimonoenergetic neutrons from 20 to $35 \mathrm{MeV}$ energies was build up to complete the integral validation experiments by differential measurements.

Extensive validation experiments of activation cross sections are carried out on IFMIF-relevant materials and on dosimetry foil detectors as well.

\section{References}

1. U. Fischer, S. Simakov et al., Validated Computational Tools and Data for IFMIF Neutronic Calculations, in Proc. 6th Int. Meeting on Nuclear Applications of Accelerator Technology, June 2003, San Diego, pp. 1000-1008.

2. P. Bém et al., Fus. Eng. Design 69, 479 (2003).

3. H.J. Brede et al., Nucl. Instrum. Meth. A 274, 332 (1989).

4. S.P. Simakov et al., Development of activation foils method for the IFMIF neutron flux characterization, SOFT 24, Fus. Eng. Design (to be published); See also P. Bém et al., EFF-DOC-944 (2005); S.P. Simakov et al., EFF-DOC-951(2005).

5. Y. Uwamino et al., Nucl. Instrum. Meth. A 389, 463 (1997).

6. R.A. Forrest et al., Validation of EASY-2005 using integral measurements, UKAEA FUS 526, January 2006.

7. P. Bém et al., Fus. Eng. Design, 75-79 829 (2005); see also S.P. Simakov et al., EFF-DOC-875 (2003); P. Bém et al., EFFDOC-910 (2004); S.P. Simakov et al., EFF-DOC-913 (2004); M. Honusek et al., EFF-DOC-945 (2005); E. Šimeková et al., EFFDOC-946 (2005).

8. M. Honusek et al. (these proceedings). 